Research Article

\title{
Causes of under-five mortality using verbal autopsy and social autopsy studies (VASA) in Alexandria, Egypt, 2019
}

\author{
Ramy Mohamed Ghazy ${ }^{1}$ (D, Mohga M Fekry², Abdel-Rahman Omran ${ }^{3}$ (D), Mohamed Mostafa Tahoun ${ }^{1}$ \\ ${ }^{1}$ High Institute of Public Health, Alexandria University, Alexandria, Egypt, 2 Maternal Child Health Department, Alexandria Health Directorate, Ministry \\ of Health and Population, Alexandria, Egypt, ${ }^{3}$ Ministry of Health and Population, Alexandria, Egypt \\ Keywords: preterm delivery, communicable diseases, egypt, under-five mortality, social autopsy, verbal autopsy \\ https://doi.org/10.29392/001c.14379
}

\section{Journal of Global Health Reports}

Vol. 4, 2020

\section{Background}

Determination of death causes is an important building block for civil registration and vital statistics (CRVS). This study aimed at identifying the causes of under-five mortality (U5M) in Alexandria using verbal autopsy (VA).

\begin{abstract}
Methods
Data of 645 under-fives (452 neonates \& 193 post-neonates and children) deaths were retrieved from records from January 2018 to June 2019, care-givers were interviewed using Verbal Autopsy and Social Autopsy Studies (VASA), version 1.5.1 developed by the World Health Organization. Questionnaires (neonatal and child forms) were uploaded to the Open Data Kit collector (ODK), analyzed by Smart-VA software to identify the underlying cause of death (UCOD).
\end{abstract}

\section{Results}

The response rate to VASA was $66 \%$. Neonatal mortality, post-neonatal mortality, and child mortality represented $70.1 \%, 24.5 \%$, and $5.4 \%$ of U5M respectively. There was no statistically significant difference between males and females in cause-specific mortality fraction (CSMF), $\mathrm{P}>0.05$. The main UCODs among neonates were preterm delivery (57.3\%), congenital malformation (17.3\%), congenital pneumonia (10.8\%), birth asphyxia (8.4\%), and stillbirth (3.1\%). The most-reported UCODs among post-neonates and children were pneumonia (25.9\%), childhood cardiovascular diseases (22.8\%), digestive system diseases (11.9\%), and diarrhea and dysentery (7.8\%). The main UCODs among under-five children were preterm delivery $(40.2 \%)$, followed by congenital malformation (12.1\%), pneumonia (7.8\%), congenital pneumonia (7.6\%), childhood cardiovascular diseases (6.8\%), and birth asphyxia (5.9\%). Communicable diseases, maternal, neonatal, and nutritional diseases caused $72.0 \%$ of U5M, while non-communicable diseases and injuries caused $25.7 \%$ and $2.4 \%$ of all U5M, respectively.

\section{Conclusions}

VASA declared that communicable diseases, maternal, nutritional, and neonatal diseases remain the main killer of $\mathrm{U} 5 \mathrm{C}$ in Alexandria.

Under-five mortality trends (U5M) are an important benchmark for a country's health status and progress towards the achievement of the sustainable development goals (SDGs). ${ }^{1}$ It worth noting that over the last decades, all countries have made very rapid progress against child mortality. From 1800 to 1950 global mortality has halved from around $43 \%$ to $22.5 \%$. In the same context, the child mortality rate declined five-folds to reach $4.5 \%$ from 1950 to 2015 . All countries have benefitted from this marvelous worldwide decline in child mortality rates. ${ }^{2}$ By the year 2030, the target of SDGs is to reduce preventable mortali- ties among neonates to be as low as 12 per 1000 live births and to reduce U5M to be as low as 25 per 1000 live births. ${ }^{3}$

While the global burden of children's deaths remains high, children's chances of survival vary dramatically across regions and countries; for many under-five children (U5C), the socioeconomic status and the geographic nature of their birth-place determine their access to high quality and affordable health care. ${ }^{4}$ Therefore, a large number of U5C continue to die from easily preventable and treatable diseases or conditions because the world has failed to provide their basic rights for survival and health. ${ }^{5}$ First most and foremost, efforts must be made to work together to save 
children live as the current trends predict that around 52 million U5C will die between 2019 and 2030. ${ }^{6}$

In Egypt, the infant mortality rate is $22 / 1,000$ live births, while the neonatal mortality rate is $14 / 1,000$ live births. Contrasting these rates with the overall level of U5M rate (27/1,000 live births) indicates that almost $80 \%$ of early U5M take place before a child's first birthday, with half of these deaths occurring during the first month of life. ${ }^{6}$ These rates were lower than what was reported by the Egyptian Demographic Health Survey in 2008, as both the U5M and neonatal mortality rates were 33.4 and 17.5 per 1,000 live births, respectively. ${ }^{7}$ This reduction in mortality indicators signifies the great improvement in the maternal and child health services since $1990 .^{8}$

Despite sizeable efforts made to reduce the U5M, critical data about the main causes of death are still lacking. ${ }^{9}$ In 2016, the Eastern Mediterranean Regional Office reported that the most common causes of U5M were prematurity (20.6\%), congenital anomalies (11.6\%), birth asphyxia (9.8\%), and pneumonia (9.7\%). Indeed, in low and middleincome countries (LMIC), causes of U5M death are frequently inaccurately registered by the civil registration and vital statistics (CRVS), and nearly half of these countries fail to meet the United Nations standards for death registration. ${ }^{10}$ Surprisingly, the good quality of data related to recording the actual cause-of-death is lacking for $65 \%$ of the world's population. Inadequate data on cause-specific mortality patterns impede the development of sound health policies, planning, monitoring, and evaluation. Accurate and complete quality data concerning the causes of $\mathrm{U} 5 \mathrm{M}$, have to be available to support resource allocation, planning and evaluating of different public health interventions. ${ }^{11}$

Although complete vital registration systems with an accurate medical certification of the causes of death are considered the best source of mortality data, particularly U5M data, yet these systems are challenging to be implemented, in terms of the needed human, non-human and financial resources, ${ }^{12}$ especially in large populations including Egypt. In Alexandria, the quality of registration of U5M especially those occurring at home was poor. ${ }^{13}$ In areas where medical certification of cause of death is not possible and CRVS systems are weak, verbal autopsy (VA) has been introduced as a practical method for determining the probable cause of death. VA consists of a retrospective interview on the signs and symptoms of the fatal illness with the mother or other caregiver of the child. ${ }^{14}$ This is done using a standardized questionnaire that elicits information on signs, symptoms, medical history, and circumstances preceding death. VA is now widely used in LMIC to determine cause-specific mortality, and it is increasingly being used for disease surveillance and in sample registration systems. (15) In an attempt to further standardize the VA cause of death (COD) assignment and alleviate physician time and costs, efforts have been made to automate VA using various computational algorithms. The current leading computational VA techniques include InterVA-4, Tariff, InSilicoVA, King-Lu, and Naïve Bayes Classifier. To the extent of the researcher's knowledge, no studies in Egypt used au- tomated VA to identify the causes of U5M. For that reason, this study was conducted in Alexandria where COD is not properly identified to address the causes of U5M using Verbal Autopsy and Social Autopsy Studies (VASA), version 1.5.1 developed by the World Health Organization (WHO).

\section{METHODS}

\section{STUDY SETTING}

The study was conducted in Alexandria Governorate, the second-largest Egyptian city. The city extends about 32 $\mathrm{km}$ along the Mediterranean Sea on the north-west coast of Egypt. Alexandria is divided into eight administrative health districts, namely; Sharq, Gharb, Wasat, Gomrok, Amriya, Agamy, Montaza, and Borg El-Arab containing 40 health offices. Data on registered deaths were collected from 20 randomly selected health offices (Figure 1).

\section{STUDY POPULATION}

All registered U5M from January 2018 to June 2019 were identified form available databases in the selected health offices. A total of 3064 deaths were identified, and 1382 deaths had incorrect or incomplete data. Out of the remaining 1682, 980 cases were traced randomly until the required sample size was collected; 645 relatives completed the VA questionnaire.

\section{SAMPLE SIZE CALCULATION}

Assuming that the mean population of each selected cluster is 1500 child/cluster, the U5MR estimate of $21 / 1000$, a total of 20 clusters were randomly selected, the proportion of U5M occurring within the health facility are $10 \%$ of all recorded deaths, and the under-notification rate is $1 \%$, the minimal required VA per year would be estimated as 630 (power is $80 \%$ and alpha error is 0.05 ). The sample size was calculated using CRVS-VA Sample Size Calculator Tool v 1.0 .

\section{DATA COLLECTION}

The address of the decedent was obtained from the health office records, and trained field investigators, blinded to the cause of death from either the routine system or the expert review, conducted the interviews using the 2016 WHO Verbal Autopsy Instrument v1.5.1 tool both neonate and child versions. ${ }^{15}$ The mortality indicators presented in this study were neonatal mortality, infant mortality, and U5M.

The questionnaires were translated into Arabic by two groups of bilingual experts, followed by backward translation by a native English speaker. The Arabic tool was uploaded to an open data kit (ODK) collector. A pilot test $(n=30)$ was conducted to assure clarity of the questions, local perceptions of specific symptoms and signs, accuracy and completeness of questionnaires, and to estimate the time needed to complete the questionnaire. VA was completed by 18 trained interviewers in a period of 6 months (starting May through October 2019). All surveyors and supervisors received special training on conducting the VA 


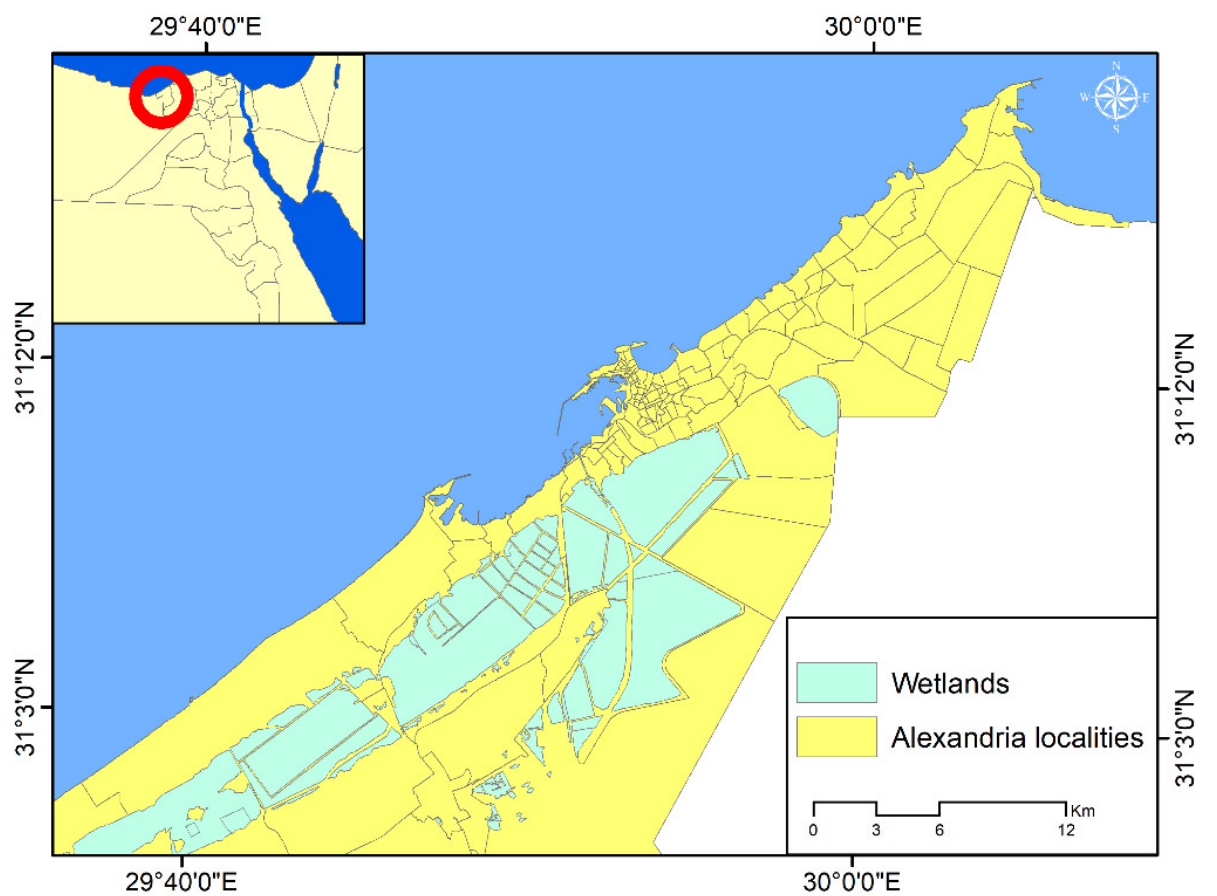

Figure 1. A map depicting Alexandria's eight districts.

interview. The training workshops were held at the High Institute of Public Health (HIPH) to familiarize the team members with the data collection tool, ensure their ability to use the mobile application and train them on how to deal with the psychological issues that may arise during the interview and hinder data collection. All surveyors were public health workers and on average, the interview required about 20 minutes. Causes-of-death classification was based on the International statistical classification of diseases and related health problems, tenth revision (ICD-10) which is the mandatory level of coding for international reporting to the WHO mortality database. It has 21 chapters and 2046 categories of diseases, syndromes, external causes, or consequences of the external causes. ${ }^{16}$ Data were extracted to Excel worksheets from the ODK server and were fed into the statistical software. Data analysis was done using Statistical Package for Social Sciences (SPSS) version 25 and Smart VA (Tariff 2.0) diagnostic algorithm. Smart VA is compatible for use with the WHO 2016 VA instrument and can be used in CRVS. Tariff method is an approach that balances data-driven machine-learning methods with a level of interpretability necessary for methodological acceptance (this balance is referred to as "explainable artificial intelligence”). The Tariff method produces a set of tariff scores for each disease-symptom pair, which can be interpreted as a robust analog to a $\mathrm{z}$-score and indicate the relatedness of each symptom to each cause. ${ }^{17}$ The input of the software used in VASA analysis was either WHO-VASA neonatal ( 0 -28 days), child (28 days- 12 years), or adult (>12 years) forms while the output is graphs and tables illustrating the individual cause of death, cause-specific mortality fraction (CSMF) for each group and the global burden of disease broad categories (GBD). In this study, we will present the CSMF and global burden of disease broad categories

\section{ETHICS APPROVAL}

The Ethics Committee of the HIPH and the Central Directorate for Research and Health Development approved this study. Written informed consent was obtained from all interviewees after the explanation of the purpose of the study and assurance of confidentiality and anonymity of the collected data.

\section{RESULTS}

Based on proportional allocation; the highest participation was from Montaza district (24.8\%) followed by Wasat (14.7\%), Sharq (14.0\%), Agamy (11.6\%), and Gharb (10.9\%). The least number was sampled from Borg El- Arab (7.8\%) and Gomrok (4.3\%). Of surveyed cases, 90.5\% (584) occurred at a health care facility, $8.4 \%$ (54) of deaths occurred at home, and 1.1\% (7) occurred outside the home (road traffic accident and drowning).

Table 1 highlights the age and sex of deceased children analyzed in VASA; the number of total deaths was higher among males (331, 51.3\%) than females (314, 48.7\%). Early neonatal mortality represented $42.9 \%$ of the U5M; [143 males (51.6\%) \& 134 females (48.6\%)]. Late neonatal deaths (8-28 days) represented $27.1 \%$ of U5M; [90 males (51.4\%) \& 85 females (48.6\%)]. Post-neonatal deaths represented $24.5 \%$ of U5M; [males: 76 (48.1\%) \& females: 82 (51.9\%)]. Of U5M, a total of 35 (5.4\%) children died between 1-5 years [22 (62.9\%) among males and 13 (37.1\%) among females]. These differences of deaths at different ages were not statistically significant between males and females (Chisquare, $\chi^{2}=2.53, P=0.47$ ) 
Table 1. Age-sex distribution of VASA cases, Alexandria, Egypt, 2019

\begin{tabular}{|c|c|c|c|c|c|c|c|}
\hline $\begin{array}{l}\text { Age at } \\
\text { death }\end{array}$ & $\begin{array}{c}\text { Total } \\
(n=645)\end{array}$ & $\begin{array}{c}\text { Early } \\
\text { neonate }^{1} \\
(n=277)\end{array}$ & $\begin{array}{c}\text { Late } \\
\text { neonate }^{2} \\
(n=175)\end{array}$ & $\begin{array}{l}\text { Post-neonate } \\
\text { infant }^{4}(n=158)\end{array}$ & Child $^{5}(n=35)$ & $\begin{array}{c}\text { Chi- } \\
\text { square, } \\
\mathrm{X}^{2}\end{array}$ & $\begin{array}{c}\text { P- } \\
\text { value }\end{array}$ \\
\hline Males & $\begin{array}{c}331 \\
(51.3)\end{array}$ & $143(51.6)$ & $90(51.4)$ & 76 (48.1) & 22 (62.9) & \multirow[b]{2}{*}{2.531} & \multirow[b]{2}{*}{0.060} \\
\hline Females & $\begin{array}{c}314 \\
(48.7)\end{array}$ & $134(48.4)$ & 85 (48.6) & 82 (51.9) & 13(37.1) & & \\
\hline
\end{tabular}

${ }^{1}$ death $<7$ days, ${ }^{2}$ death7-28 days, ${ }^{3}$ death after 28 days and before 1 year; ${ }^{4}$ death before 1 year; ${ }^{5}$ death between $1-5$ years.

\section{CAUSE-SPECIFIC MORTALITY FRACTION ANALYSIS (CSMF)}

Table 2 illustrates the main causes of neonatal mortality by VASA; preterm delivery caused $57.3 \%$ (259 cases) of all neonatal mortalities; (52.9\% males and $47.1 \%$ females), followed by congenital malformation $17.3 \%$ (78 cases), with a higher prevalence among females than males $(53.8 \%$ and $46.2 \%$, respectively). Congenital pneumonia killed 49 neonates (10.8\%), with a higher proportion of males affected than females (59.2\% vs $40.8 \%)$. Birth asphyxia caused $8.4 \%$ (38 cases) of neonatal mortality, with higher proportion among females (60.5\%) than males (39.5\%). Stillbirth resulted in $3.1 \%$ of neonatal deaths; an equal number of stillbirths identified among males and females (7 for each group). However, these mentioned differences in cause-specific mortality fraction were not statistically significant between males and females $\left(X^{2}=4.64, P=0.46\right)$.

The most commonly reported causes of post-neonatal and child mortality by VASA were pneumonia $25.9 \%$ (50 cases); more females were affected (68.0\%) than males (32.0\%). Cardiovascular diseases ordered as the second cause of death; $22.8 \%$ (44 cases), with nearly equal proportion among males (52.3\%) and females (47.7\%), digestive diseases caused $11.9 \%$ of deaths (23 cases) with an equal distribution like childhood cardiovascular diseases. Diarrheal diseases and dysentery resulted in $7.8 \%$ (15 cases) of deaths (46.7\% among males and $53.3 \%$ among females). It is worth mentioning that difference in cause-specific death was not statistically different between males and females $\left(\chi^{2}=8.23, P=0.412\right)$.

Figure 2 illustrated the main causes of U5M; preterm delivery resulted in 259 (40.2\%) of all deaths $(21.2 \%$ and $18.9 \%$ among males and females respectively, followed by congenital malformation 78 cases (12.1\%) about 5.6\% of male deaths were due to congenital malformation while it was incriminated in $6.5 \%$ of female deaths. Pneumonia was the third killer of U5C; 50 children (7.8\%) died due to pneumonia; with a higher incidence among females (5.3\%) than males (2.5\%). Congenital pneumonia ordered as the 4 th cause; a total of 49 (7.6\%) U5C died due to congenital pneumonia with a higher prevalence among females (4.5\%) than males (3.1\%). Childhood cardiovascular diseases are the $5^{\text {th }}$ common cause of U5M; they caused $6.8 \%$ (44 cases) of all U5M; 3.6\% (23 cases) among males and 3.3\% (21 cases) among females. Birth asphyxia is the $6^{\text {th }}$ cause of U5M; it resulted in 37 (5.9\%) deaths among U5C with a higher proportion among females (3.6\%) than males (2.3\%).

\section{GLOBAL BURDEN OF DISEASE BROAD CATEGORIES (GBD)}

Smart-VA analysis of the classification of COD by the global burden of disease board categories revealed that communicable diseases, maternal, neonatal, and nutritional diseases caused $71.9 \%$ of U5M. Non-communicable diseases represented $25.7 \%$ of all deaths and lastly, injuries represented $2.4 \%$.

\section{INDIVIDUAL CAUSE OF DEATH}

Notably, individual case analysis revealed that $23.0 \%$ of cases had an undetermined cause of death. This outcome was not illustrated in this study; it will be used to validate the diagnostic validity of VASA.

\section{DISCUSSION}

Identifying the current causes of U5M within a specific period in communities is essential to create a solid ground on which health authorities can foresee future death-scenarios. Consequently, authorities would effectively plan health services and strategies. In developing countries, many deaths take place outside health facilities, this shocking fact makes the recording of the actual COD is not always accurate. Different approaches are being used to drive the COD as defined in ICD-10 like physician review, predefined expert algorithm, and data-driven algorithm. This research aimed at identifying COD among U5C in Alexandria by using Smart VA software to analyze data collected by VASA v1.5.1.

In the current research, the data collector team contacted 980 relatives until fulfilling the required sample size. The total number of interviewed relatives was 645 . The calculated response rate was $66 \%$. Non-responders include either those who refused to make the interview or those who did not attend the interview after their initial acceptance. The response rate was considered satisfactory as the concept of the verbal autopsy may be new to the Egyptian communities, including health care workers. Notably, the interviewees thought that the research team was investigating medical malpractice, many of them were motivated to respond to the research team, others responded to the research because of a criminal assault related to some included deaths. On the other hand, in rural areas of Tanzania, higher response rates (83\% and $89 \%$ ) were reported by Mwanyangala et al., ${ }^{18}$ in two surveys conducted 
Table 2. Causes of neonatal and child mortality using VASA analyzed by Smart-VA, Alexandria, Egypt, 2019

\begin{tabular}{|c|c|c|c|}
\hline \multicolumn{4}{|c|}{ Causes of neonatal Deaths using VASA analyzed by smart VA } \\
\hline Cause (ICD10) & Total $(n=452)$ & Males ( $n=232)$ & Females $(n=220)$ \\
\hline & No (\%) & No (\%) & No (\%) \\
\hline Stillbirth (P95) & $14(3.1)$ & $7(50.0)$ & $7(50.0)$ \\
\hline Neonatal Meningitis/Sepsis (P36) & $14(3.1)$ & $8(57.1)$ & $6(42.9)$ \\
\hline Birth asphyxia (P21) & $38(8.4)$ & $15(39.5)$ & $23(60.5)$ \\
\hline Congenital Pneumonia (P23) & $49(10.8)$ & $29(59.2)$ & $20(40.8)$ \\
\hline Congenital malformation (Q89) & 78(17.3) & $36(46.2)$ & $42(53.8)$ \\
\hline Preterm Delivery (P07) & $259(57.3)$ & $137(42.9)$ & $122(57.1)$ \\
\hline
\end{tabular}

Degree of freedom $=5$, Chi-square $x^{2}=4.64, P=0.460$

Causes of child (aged 1 month to 5 years) deaths using VASA, analyzed by smart VA

\begin{tabular}{lccc}
\hline Cause (ICD 10) & Total (n=193) & Males (n=97) & Females (n=96) \\
\hline & No (\%) & No (\%) & $1(50.0)$ \\
Encephalitis (G04) & $2(1.0)$ & $1(50.0)$ & $1(50.0)$ \\
Hemorrhagic fever (A99) & $2(1.0)$ & $1(50.0)$ & $1(50.0)$ \\
Poisonings (X49) & $2(1.0)$ & $1(50.0)$ & $1(33.3)$ \\
Sepsis (A41) & $3(1.6)$ & $2(66.7)$ & $1(33.3)$ \\
Drowning (W74) & $3(1.6)$ & $2(66.7)$ & $2(50.0)$ \\
Road Traffic (V89) & $4(2.1)$ & $3(60.0)$ \\
Measles (B05) & $5(2.6)$ & $2(40.0)$ \\
Other Infectious Diseases (B99) & $5(2.6)$ & $2(50.0)$ & $2(33.3)$ \\
Falls (W19) & $6(3.1)$ & $3(60.0)$ & $2(33.3)$ \\
Childhood Cancer (C76) & $6(3.1)$ & $4(66.7)$ & $2(20.0)$ \\
Meningitis (G03) & $10(5.2)$ & $4(66.7)$ & $4(31.8)$ \\
Other Defined Causes of Child Deaths (UU2) & $13(6.7)$ & $8(80.0)$ & $8(53.3)$ \\
Diarrhea/Dysentery (A09) & $15(7.8)$ & $9(69.2)$ & $11(47.7)$ \\
Digestive Diseases (K92) & $23(11.9)$ & $7(46.7)$ & $21(47.7)$ \\
Childhood Cardiovascular Diseases (I99) & $44(22.8)$ & $12(52.3)$ & $34(68.0)$ \\
Pneumonia (J22) & $50(25.9)$ & $23(52.3)$ & $16(32.0)$ \\
\hline
\end{tabular}

5 years apart (2002 and 2007). On the same vein, RamirezVillalobos et al. ${ }^{19}$ reported a higher response rate $(95.8 \%)$ in a study conducted in Mexico. The objective of their study was to investigate the cause of death among 1198 deaths from all age groups, where the number of infant and toddler deaths was 75 cases $(6.26 \%)$ this might have rendered the interview easier than in the case of infants and children deaths. Similarly, Allotey et al. ${ }^{20}$ reported a very high response rate (99.5\%) to VA; however, the latter study may be considered a pilot study as the total number of investigated cases was 66 subjects only. This figure is considered relatively small to conduct a verbal autopsy study.

In this study, neonatal deaths represented 70.1\% (452/ 645 ) of the U5M, more specifically, early neonatal mortality (within the first week of life) represented 61.3\% (277/452) of neonatal mortality, and late neonatal mortality represented 38.7\% (175/452). Post-neonatal mortality and child mortality represented (24.5\%) and (5.4\%) respectively. The neonatal deaths as a percentage of U5M are higher than the figure reported by UNICEF (52.9\%), this difference may be due to the relatively small sample size of deaths required in the verbal autopsy analysis, besides, not all governorates were represented in this research. Also, Alexandria governorate is classified as an urban governorate, while other Egyptian governorates that have a higher presentation of rural districts were not studied; this difference in localities may be an attribute to increased risk of U5M. ${ }^{21}$

The total number of deaths was slightly higher among males than females (331 vs 314). Based on smart VA analysis; the main causes of neonatal mortality were preterm delivery accused of (57.3\%) followed by congenital malformation (17.3\%), neonatal pneumonia (10.8\%), and birth asphyxia (8.4\%). On the same line, Campbell et al., ${ }^{22}$ reported that the main causes of neonatal mortality were prematurity, birth asphyxia, infection, and congenital malformation. The most-reported causes of post-neonatal and child mortality were pneumonia (25.9\%), childhood cardiovascular diseases (22.8\%), digestive diseases (11.9\%), diarrheal diseases, and dysentery (7.8\%). The main causes of U5M in this research were preterm delivery (40.2\%) followed by 


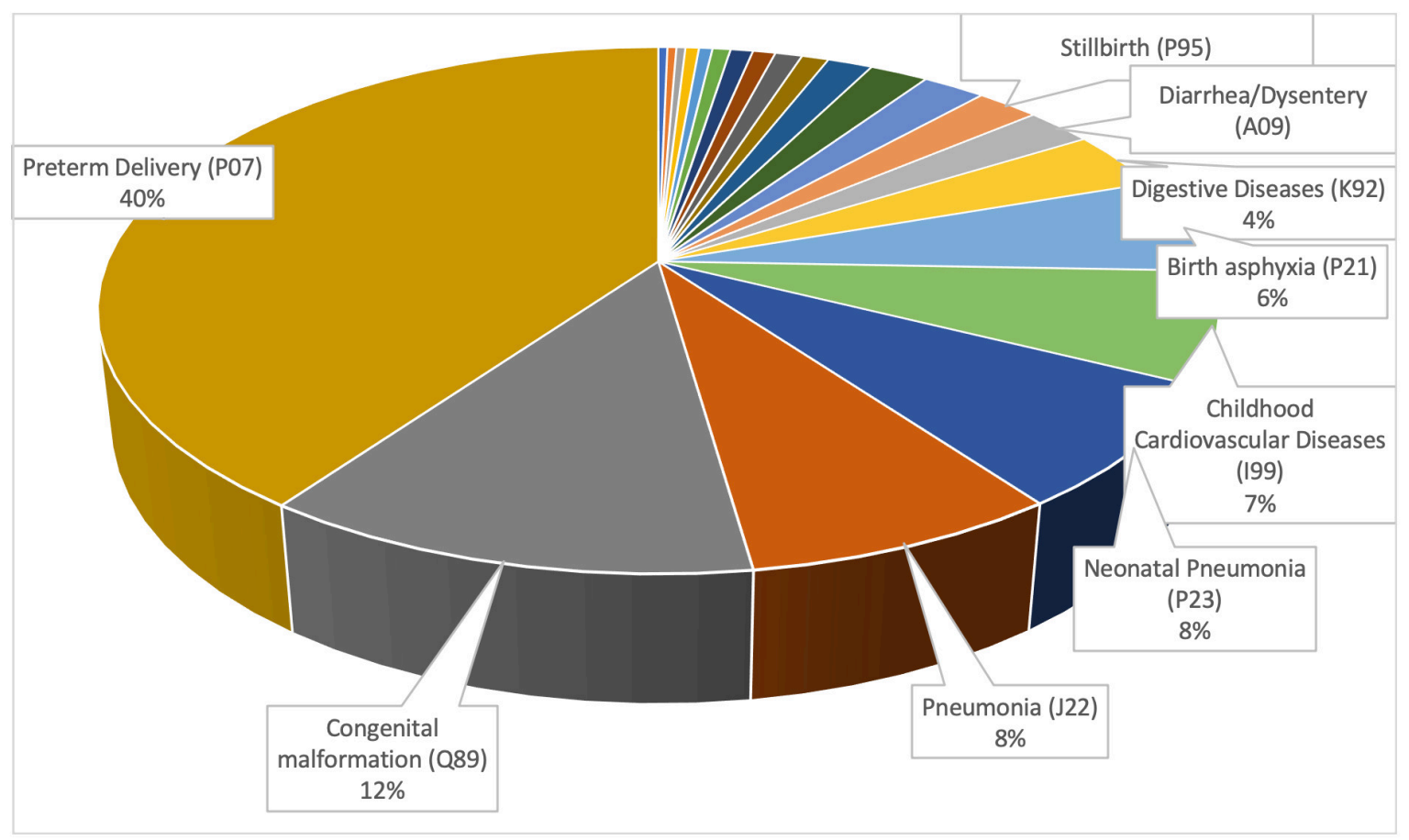

Figure 2. The main identified causes of under-five mortality using verbal autopsy, Alexandria, Egypt, 2019.

congenital malformation (12.1\%), pneumonia (7.8\%), neonatal pneumonia (7.6\%), and childhood cardiovascular diseases (6.8\%). These results were similar to what was reported by WHO-EMRO; the most reported causes of death among under-five were prematurity, pneumonia, congenital malformations. ${ }^{23}$

In this work, the percentage of the undetermined causes of death was $23.0 \%$. It slightly exceeded the figure reported by Ramirez-Villalobos et al., ${ }^{19}$ a total of $18.0 \%$ of causes were not identified (9\% in adults, $2 \%$ in neonates and $7 \%$ in children). On the same vein, Mwanyangala et al., ${ }^{18}$ reported that $33.0 \%$ of infants and neonates had an undetermined cause of death. This inconvenience in the diagnosis of the cause of death may be due to insufficient data obtained from interviewed relatives. Another explanation for the unidentified cause was that $9 \%$ of deaths occurred at homes this may have contributed to the missed clinical data. The total number of individual COD diagnosed as undetermined is 148 cases. As mentioned, Smart VA facilitates two types of analysis; individual-cause and CSMF. Unlike the individual-cause report of Smart VA analysis, in CSMF there are no 'Undetermined' causes of death. This is because Smart VA-Analyze redistributes the VAs with undetermined COD using a combination of two methods. ${ }^{24}$ Firstly, a VA with undetermined COD is fractionally distributed amongst all VA causes with weights proportional to the likelihood that the particular cause was assigned to undetermined in the gold standard database. Secondly, this fractional redistribution weight is averaged with a proportional redistribution weight selected according to the Global Burden of Disease age and sex COD distribution for the country. Based on the output report of Smart VA, the cause of undetermined death is that certain causes of death (such as pneumonia) have an inherent more difficulty to be diagnosed using VA methods than a cause like Road traffic accident.

Communicable and nutritional diseases were on top of the list of the causes of U5M, they caused $71.9 \%$ of U5M, followed by NCDs (25.7\%), and injuries (2.4\%). On the same line, Boutayeb et al,. ${ }^{25}$ reported that communicable diseases, such as diarrheal diseases and acute respiratory infections along with malaria, constituted the main killers for the U5C in developing countries located in Africa and Mediterranean region. This is mainly due to illiteracy, poverty, lack of access to health services, low vaccination coverage rates, following unhealthy traditions and customs, and poor housing conditions.

\section{STRENGTHS AND LIMITATIONS}

To the best of our knowledge, the research presented here is the first to address the causes of U5M in Egypt using electronic data collection using ODK and automated analysis of VA questionnaires using Smart-VA software. The sampling technique was another point of strength as all districts of Alexandria were proportionally represented in the population sampling.

One of the main barriers against the implementation of VA by national registration systems is the time and cost needed for data collection. Another problem was that collection of data from interviewees is a heart-rending event; parents may refuse to respond to the questionnaire, or they may not complete the interview so that a minimum duration of four weeks was required to allow adequate mourning. Even after adequate training, the minimum required time for completing the questionnaire was about 20-30 minutes; this duration was very long especially with the intention of the interviewer to elicit interviewees to mem- 
orize these painful events. Some of the respondents lost their interest, however, all of them completed the interview. Lastly, insufficient or incomplete data, like telephone number of the death notifier, date of child death or birth, or incomplete or wrong address of the case reported, were the main barriers, as we could not access these cases.

\section{CONCLUSIONS}

This study provides an evidence that interviewing deceased relatives using a VA questionnaire and smart algorithm for diagnosis of causes of death can provide information on priority health problems in resource-poor communities. We encourage the use of VA in all deaths occurring outside the health facilities. Training of the health officers responsible for registering death certificates is mandatory with an initial focus on the basic demographic data related to the deceased such as address, phone number, and relationship with the deceased. Future studies could fruitfully explore the effectiveness of the nation-wide implementation of VASA in Egypt, especially among deaths occurring outside the health facilities. Also, the quality of death registration and completeness of data should be assessed. We recommend WHO to develop other forms that address causes of deaths among different age categories like post-neonatal and children.
Acknowledgments: No words would be sufficient to express our gratefulness and gratitude towards the guardians of the deceased children who showed great enthusiasm and willingness to complete the interview despite their hard feelings. Without their support and cooperation, this work would have not been accomplished.

Funding: The author(s) disclosed receipt of the following financial support for the research, authorship, and/or publication of this article: This research was funded by the WHO/EMRO Special Grant for Research in Priority Areas of Public Health, (RPPH 18-83).

Authorship contributions: All authors contributed to the study design, and writing of the draft and final versions of the manuscript.

Competing interests: The author completed the Unified Competing Interest form at www.icmje.org/coi disclosure.pdf (available upon request from the corresponding author), and declare no conflicts of interest.

\section{Correspondence to:}

Ramy Mohamed Ghazy

MB.B.Ch, M.Sc, MPH, Dipl.Dat.Analysis, DrTH (candidate)

Assistant Lecturer of Tropical Health

High Institute of Public Health

Alexandria University, Egypt.

Postal code: 21561

ramysarah@alexu.edu.eg

https://orcid.org/0000-0001-7611-706X 


\section{REFERENCES}

1. Wang H, Bhutta ZA, Coates MM, et al. Global, regional, national, and selected subnational levels of stillbirths, neonatal, infant, and under-5 mortality, 1980-2015: A systematic analysis for the Global Burden of Disease Study 2015. Lancet. 2016;388(10053):1725-1774.

2. Roser M, Ritchie H, Dadonaite B. Child and Infant Mortality. Published online 2020. https://ourworldind ata.org/child-mortality

3. World Health Organization. Sustainable Development Goal 3: Health. Published online 2020. https://www.who.int/topics/sustainable-developmen t-goals/targets/en/

4. World Health Organization. Better Health for Poor Children: A Special Report. World Health Organization; 2001.

5. World Health Organization. Children: reducing mortality. https://www.who.int/news-room/fact-shee ts/detail/children-reducing-mortality

6. UNICEF. Levels and Trends in Child Mortality. Published online 2019. https://data.unicef.org/resour ces/levels-and-trends-in-child-mortality/

7. El-Zanaty F, Way A. Egypt Demographic and Health Survey 2008. Ministry of Health/Egypt, El-Zanaty and Associates/Egypt, and Macro International; 2009.

8. Kassebaum NJ, Barber RM, Bhutta ZA, et al. Global, regional, and national levels of maternal mortality, 1990-2015: A systematic analysis for the Global Burden of Disease Study 2015. Lancet. 2016;388(10053):1775-1812.

9. Yaya S, Uthman OA, Okonofua F, Bishwajit G. Decomposing the rural-urban gap in the factors of under-five mortality in sub-Saharan Africa? Evidence from 35 countries. BMC Public Health. 2019;19(1). do i:10.1186/s12889-019-6940-9

10. World Health Organization. Child mortality rates plunge by more than half since 1990 but global MDG targets missed by a wide margin. Published online 2020.

11. Rerimoi AJ, Jasseh M, Agbla SC, Reniers G, Roca A, Timæus IM. Under-five mortality in The Gambia: Comparison of the results of the first demographic and health survey with those from existing inquiries. Masquelier B, ed. PLoS ONE. 2019;14(7):e0219919. do $\mathrm{i}: 10.1371 /$ journal.pone.0219919
12. Joubert J, Rao C, Bradshaw D, Dorrington RE, Vos T, Lopez AD. Characteristics, availability and uses of vital registration and other mortality data sources in post-democracy South Africa. Glob Health Action. 2012;5(1):1-19. doi:10.3402/gha.v5i0.19263

13. Gihan I, Gewaifel MMA. Assessment of Quality of Registration of Causes of Death at Home using Verbal Autopsy in Alexandria, Egypt. EJCM. 2019;37(3):45-54. doi:10.21608/ejcm.2019.43369

14. Nichols EK, Byass P, Chandramohan D, et al. The WHO 2016 verbal autopsy instrument: An international standard suitable for automated analysis by InterVA, InSilicoVA, and Tariff 2.0. PLoS Med. 2018;15(1):e1002486. doi:10.1371/journal.pme d.1002486

15. World Health Organization. Verbal autopsy standards: Ascertaining and attributing causes of death. Published online 2020. https://www.who.int/h ealthinfo/statistics/verbalautopsystandards/en/

16. World Health Organization. ICD-10: International Statistical Classification of Diseases and Related Health Problems : Tenth Revision. 2nd ed. World Health Organization

17. Nichols EK, Byass P, Chandramohan D, et al. The WHO 2016 verbal autopsy instrument: An international standard suitable for automated analysis by InterVA, InSilicoVA, and Tariff 2.0. PLoS Med. 2018;15(1):e1002486. doi:10.1371/journal.pme d.1002486

18. Mwanyangala MA, Urassa HM, Rutashobya JC, et al. Verbal autopsy completion rate and factors associated with undetermined cause of death in a rural resource-poor setting of Tanzania. Popul Health Metrics. 2011;9(1):41. doi:10.1186/1478-7954-9-41

19. Ramirez-Villalobos D, Stewart AL, Romero M, Gomez S, Flaxman AD, Hernandez B. Analysis of causes of death using verbal autopsies and vital registration in Hidalgo, Mexico. Joshi R, ed. PLoS ONE. 2019;14(7):e0218438. doi:10.1371/journal.pon e. 0218438

20. Allotey PA, Reidpath DD, Evans NC, et al. Let's talk about death: Data collection for verbal autopsies in a demographic and health surveillance site in Malaysia. Glob Health Action. 2015;8(1):28219. doi:1 $\underline{0.3402 / \text { gha.v8.28219 }}$ 
21. UNICEF. Cross-sector Indicators Geographic Area: Egypt. Published online 2020. Accessed May 6, 2020. https://data.unicef.org/resources/data_explorer/ unicef_f/?ag=UNICEF \&df=GLOBAL_DATAFLOW \&ve $\mathrm{r}=1.0 \& \mathrm{dq}=\mathrm{EGY} . \mathrm{CME}$ TMYOT4+CME TMY0+CME TM M0+CME_TMY1T4+CME_PND+CME_MRY1T4+CM E_MRY0T4+CME_MRY0+CME_MRM0. T.\&startPerio $\underline{\mathrm{d}=2016 \text { \&endPeriod }=2019}$

22. Campbell O, Gipson R, El Mohandes A, et al. The Egypt national perinatal/neonatal mortality study 2000. J Perinatol. 2004;24(5):284-289. doi:10.1038/sj.j p.7211084
23. World Health Organization. Child and adolescent health. Published online 2020. Accessed May 3, 2020. http://www.emro.who.int/child-adolescent-health/da ta-statistics/egypt.html

24. Serina P, Riley I, Stewart A, et al. Improving performance of the Tariff Method for assigning causes of death to verbal autopsies. BMC Med. 2015;13(1):1-13. doi:10.1186/s12916-015-0527-9

25. Boutayeb A, Serghini M. Health indicators and human development in the Arab region. Int J Public Health. 2006;geographics.5(1):61. 